\title{
Traduire
}

Une autre perspective sur r tr traduction

Revue française de la traduction

$222 \mid 2010$

Traduire pour le théâtre

\section{Entretien avec William Nadylam}

Traduire et mettre en scène Stuff Happens ou le réalisme anglo-saxon à l'épreuve de la scène française

Julie de Faramond

\section{OpenEdition}

1 Journals

Édition électronique

URL : https://journals.openedition.org/traduire/438

DOI : $10.4000 /$ traduire.438

ISSN : 2272-9992

Éditeur

Société française des traducteurs

Édition imprimée

Date de publication : 15 juin 2010

Pagination : 38-41

ISSN : 0395-773X

\section{Référence électronique}

Julie de Faramond, «Entretien avec William Nadylam », Traduire [En ligne], 222 | 2010, mis en ligne le

12 novembre 2013, consulté le 21 septembre 2021. URL : http://journals.openedition.org/traduire/438 ; DOI : https://doi.org/10.4000/traduire.438

Ce document a été généré automatiquement le 21 septembre 2021. 


\section{Entretien avec William Nadylam}

Traduire et mettre en scène Stuff Happens ou le réalisme anglo-saxon à l'épreuve de la scène française

\section{Julie de Faramond}

\section{NOTE DE L'ÉDITEUR}

Recueilli et présenté par Julie de Faramond

Stuff Happens de David Hare

Mise en scène de Bruno Freyssinet et William Nadylam

Traduction en français de William Nadylam

Création à Nanterre-Amandiers, le 13 mai 2009

1 Traduire et monter en français Stuff Happens, de l'auteur britannique David Hare représentait une gageure. Célébré en Angleterre comme auteur d'une œuvre théâtrale de qualité, connu du grand public par ses pièces et ses scénarios à succès (comme celui de The Reader, nominé aux Golden Globes en 2008), anobli par la reine, David Hare est fort loin de la figure de l'auteur étranger, incompris en son pays, que le théâtre français aime à accueillir en son sein. En premier lieu, le choix de faire référence à l'histoire récente (le 11 septembre et le déclenchement de la seconde guerre du Golfe) et à son traitement audiovisuel de manière à l'intégrer telle qu'elle nous fut donnée à voir et à entendre dans un théâtre verbatim est tout à fait étranger à notre tradition scénique. En second lieu, le mélange entre deux univers culturels (low et high culture), opéré avec une certaine ingénuité, détonnait sur une scène subventionnée. En revanche, la clarté du propos et de la mise en scène de William Nadylam et Bruno Freysinnet, la qualité des comédiens, furent un atout qui a permis à Stuff Happens de toucher son public et d'être largement médiatisé. Comme par une ultime ironie, les media audiovisuels ont abondamment couvert un spectacle qui s'interrogeait précisément sur leur fonctionnement et leur impact dans l'imaginaire collectif. 
Julie de Faramond :

Écrite par le Britannique David Hare, jouée aux États-Unis et en Angleterre, Stuff Happens est une pièce qui appartient à une tradition d'écriture très différente de celle qui prévaut en France. La production, le jeu des acteurs, l'attente des spectateurs... D'Angleterre en France, la pièce est en quelque sorte passée d'un système théâtral à un autre.

William Nadylam :

Je crois à la portée universelle de la parole, à sa valeur musicale et j'aime à imaginer que lorsqu'on vient au théâtre, c'est pour la recevoir, pour l'entendre, pour la voir mise en scène, pour faire partie d'une histoire, autant que pour être touché par la vibration des percussions de la langue. Lorsque je jouais, en français, Le Cid ou Hamlet en Europe de l'Est, en Amérique du Sud ou aux États-Unis, quelque chose m'a intrigué : je me suis demandé pourquoi tous ces gens venaient m'écouter? En fait, j'ai compris que j'étais simplement le représentant de la culture française au même titre que les ballets de l'Opéra de Paris... Ils n'attachaient pas tant d'importance à ce que je racontais, à comment je l'interprétais, ils venaient juste pour entendre le français. À Venise, à Novossibirsk ou à Moscou, j'ai vu les gens avoir les mêmes réactions, sentir les mêmes choses, comme si cela se situait au-delà des mots, cela se passait entre les sons qu'émet l'acteur. C'est un des mécanismes qui m'ont fait penser que cette pièce, Stuff Happens, devait être montrée au public et qu'il fallait passer outre ces questions de transposition et de traduction.

Lorsque David Hare écrit cette pièce, il part d'une réaction d'indignation, en tant que citoyen anglais face aux guerres déclenchées par les USA et la Grande-Bretagne. Ce genre de théâtre manque en France, je trouve. Entre Anglais et Français, nos points de vue sont un peu différents, mais je ne comprends pas qu'un tel théâtre n'existe pas ici. Avant de monter cette pièce, j'avais le projet de monter Coriolan, et puis, je me suis dit «Tiens! Voici un matériau qui est moderne ». Au départ, j'ai eu un réflexe de méfiance à l'égard de l'écriture contemporaine. Et puis, je suis allé à l'encontre de ce réflexe, et j'ai décidé de ne pas attendre que cette chose soit validée, entérinée par un collège de savants... Mais je savais aussi qu'avec David Hare, je ne prenais pas un très grand risque : monté quatorze fois au National Theatre, à Londres, c'est un grand auteur de théâtre anglais.

$\mathrm{JdF}$ : II y a quand même en France un public habitué à voir les événements politiques récents traités plutôt par la voie documentaire, avec montage de textes théoriques et de témoignages, dans une tradition avant-gardiste mâtinée de post-modernisme...

W.N. : Lorsque je monte une pièce, c'est pour combler un manque que j'ai en tant que comédien par rapport à la matière que l'on me propose. Au départ, nous avions le désir de nous créer un public. Bruno Freysinnet et moi ressentions le besoin de traiter ce sujet, les événements politiques récents, de façon nouvelle, qui ne soit pas biaisée par la passion ou un préjugé. La pièce de David Hare est une dissection de quelque chose, la tentative de trouver par cette dissection la théâtralité du réel. De ce qui s'est déroulé sous nos yeux, mais qui nous a été donné à doses homéopathiques. On peut penser qu'il n'y a rien dedans qu'on ne sache déjà, la pièce ne nous apporte rien de neuf, mais le propos n'est pas d'apporter quelque fait nouveau permettant d'appréhender ces événements, mais de disséquer comment ces événements nous ont été donnés à voir. Je sais que le théâtre peut à l'occasion montrer quelqu'un qui sert à une mère ses propres enfants à manger, comme dans Titus Andronicus, ou des pièces contemporaines où l'on représente une violence qui nous éloigne de notre quotidien. Dans Stuff Happens, il s'agit d'aller chercher une violence acceptable, que tout le monde a vu et que beaucoup ont 
accepté, de passer outre cet instinct de protection qui nous pousse à éloigner de nous la figure du Mal. Cette écriture, très linéaire, très proche du réel, du verbatim, ne va pas chercher à théâtraliser à nouveaux frais ce qui a déjà été mis en scène dans le réel. Alors que Vinaver, par exemple, a redisposé le réel, l'a inscrit dans un style. Il y a de la crudité dans le théâtre anglo-saxon. Dans le théâtre germanique aussi, sauf qu'on en rajoute une dose supplémentaire : on gratte dans la plaie un petit peu plus, rendant la banalité du réel un peu plus spectaculaire.

$\mathrm{JdF}$ : Le travail de traduction vous a-t-il donné des pistes dramaturgiques, le passage d'une langue à l'autre a-t-il contribué à préparer votre mise en scène?

W.N. : J'avais des fantasmes lorsque j'ai commencé à le traduire, ce que j'ai fait uniquement parce que je voulais la monter et qu'elle n'était pas traduite. La traduction s'est faite par décantation : je n'ai pas tenté de franciser les phrases, mais de rester très proche du texte de David Hare, de garder l'étrangeté de la construction. Fort de la petite expérience que j'avais eue lorsque nous travaillions autour de la table avec Peter Brook, Jean-Claude Carrière, Marie-Hélène Estienne au moment de monter La Tragédie $d u$ Prince Hamlet, je me suis dit qu'on pouvait y aller sans complexes : il ne fallait pas que j'interprète ce que ces gens disaient, mais juste que je traduise. Ce faisant, j'ai vu ces personnages cheminer vers moi, grandir indépendamment les uns des autres, de l'histoire. Les grands traits que j'avais dessinés, sur ce que la pièce devait dire, se sont infléchis par eux-mêmes. J'ai pu voir les acteurs dire ces mots et réagir : "Mais non ça ne se dit pas comme ça, mais comme ça! » et je répondais « Mais non! Tu es en train de construire une grande phrase pour dire trois choses alors que je te propose trois morceaux de phrase qui, chacun, disent une chose différente ». Et au fur et à mesure, on s'est aperçu que les trois morceaux de phrases, trois débris de phrase, qui correspondent à trois débris d'idées, se sont télescopés pour donner quelque chose et qu'il ne fallait pas mettre d'agent de liaison, d'adjectifs, ou de subjonctifs là où il n'y en avait pas, mais les livrer de façon brute et qu'alors, le personnage surgissait de luimême. Il y a une dramaturgie qui s'est révélée par décantation. La vitesse de la narration et l'enchaînement d'un point à l'autre de la pièce se sont imposés par euxmêmes.

William Nadylam est né à Montpellier d'un père camerounais et d'une mère indienne de la Réunion. Il est acteur et metteur en scène et partage sa vie entre la France, les États-Unis et les nombreux pays où il est appelé à travailler. Il a notamment joué le rôle-titre de La Tragédie de Hamlet, mis en scène par Peter Brook aux Bouffes du Nord en 2002, dans Viol de Botho Strauss, mis en scène par Luc Bondy au Théâtre de l'Odéon en 2005, et dans Candide d'Yves Laplace, mis en scène par Hervé Loichemol au théâtre de Carouge à Genève en 2009. Acteur de cinéma, il a notamment joué dans White Material de Claire Denis, sorti en mars 2010. 


\section{AUTEUR}

\section{JULIE DE FARAMOND}

Docteur en études théâtrales (Paris Ouest - Nanterre), Julie de Faramond est chercheuse associée au Centre d'Histoire Culturelle des Sociétés contemporaines. Elle a enseigné à l'université de Franche-Comté (Besançon) et à Paul Valéry (Montpellier), à l'IES abroad et à l'IUFM de Paris. Elle est l'auteur de Pour un théâtre de tous les possibles (la revue Travail théâtral, 1970-1979), publié aux éditions de l'Entretemps (http://www.lekti-ecriture.com/editeurs/Pour-un-theatre-de-tousles.html), et poursuit ses recherches sur le spectacle et le spectaculaire du XIX siècle à aujourd'hui. 